\title{
ON COMPLEX HOMOGENEOUS SINGULARITIES
}

\author{
LÊ QUY THUONG, NGUYEN PHU HOANG LAN, AND PHO DUC TAI
}

\begin{abstract}
In this article, we consider the singularity of an arbitrary homogeneous polynomial with complex coefficients $f\left(x_{0}, \ldots, x_{n}\right)$ at the origin of $\mathbb{C}^{n+1}$, via the study of the monodromy characteristic polynomials $\Delta_{l}(t)$, and the relation between the monodromy zeta function and the Hodge spectrum of the singularity. We go further with $\Delta_{1}(t)$ in the case $n=2$. This work is based on knowledge of multiplier ideals and local systems.
\end{abstract}

\section{INTRODUCTION}

Let $f$ be a homogeneous polynomial (not necessarily reduced) of degree $d$ in $n+1$ variables with coefficients in $\mathbb{C}$, which defines a holomorphic function germ at the origin $O$ of $\mathbb{C}^{n+1}$. In general, according to [20] and [16], the Milnor fiber of $(f, O)$ is up to diffeomorphism a manifold $M=f^{-1}(\delta) \cap$ $B_{\varepsilon}$, for $B_{\varepsilon} \subset \mathbb{C}^{n+1}$ a ball of radius $\varepsilon$ around $O$ and $0<\delta \ll \varepsilon \ll 1$, which has the homotopy type of a bouquet of $\mu$ spheres of dimension $n$. Since here $f$ is a homogeneous polynomial, however, $f^{-1}(\delta) \cap B_{\varepsilon}$ is a deformation retract of $f^{-1}(\delta) \cong f^{-1}(1)$, thus we may consider $M$ as $f^{-1}(1)$. The monodromy $T: H^{*}(M, \mathbb{C}) \rightarrow H^{*}(M, \mathbb{C})$ of the singularity may be given explicitly to be the $\mathbb{C}$-linear endomorphism induced by the map $\left(x_{0}, \ldots, x_{n}\right) \mapsto\left(e^{\frac{2 \pi i}{d}} x_{0}, \ldots, e^{\frac{2 \pi i}{d}} x_{n}\right)$. It becomes classical for $f$ being an isolated homogeneous singularity at $O$ where many important invariants such as the Milnor number $\mu$, the characteristic polynomials of $T$, the signature and Hodge numbers of $M$ are computed completely in topological and algebraic methods as well as via mixed Hodge structures (cf. [21, [27]).

In the case where $f$ is a reduced homogeneous polynomial, Esnault 11] introduced a method to compute the Betti numbers, the rank and the signature of the intersection matrices of the singularity $(f, O)$, using mixed Hodge structures on cohomology groups of the Milnor fiber $M$ and the existence of spectral sequences converging to the cohomology groups, together with resolution of singularity. The work by Esnault definitely inspired the study by Loeser-Vaquié [19] of the Alexander polynomial of a reduced complex projective plane curve, where they provided a formula for the Alexander polynomial of such a curve which generalizes the previous one by Libgober [17, 18. It is likely that the approaches of Libgober in [18] and Loeser-Vaquié in [19, as well as the work by Nadel in [23, are also starting points of the studies on multiplier ideals and local systems, which were thereafter studied strongly by Esnault-Viehweg [12, Ein-Lazarsfeld [10, Demailly 7], Kollar [14, Budur [2, 4, Budur-Saito 6].

Due to the development of the theory of multiplier ideals and local systems, Budur [5] gives an explicit description of the local system of the complement in $\mathbb{P}^{n}$ of the divisor defined by a homogeneous polynomial $f$ without the condition of reducedness. In the present work, we use Budur's article [5] to study the characteristic polynomials, the Hodge spectrum and the monodromy zeta function of an arbitrary homogeneous hypersurface singularity. Let us now review in a few words what we shall do. We denote by $D$ the closed subscheme of $\mathbb{P}^{n}$ defined by the zero locus of a degree $d$ homogeneous polynomial $f$ and by $U$ the complement of $D$ in $\mathbb{P}^{n}$. Then, as shown in [4, [5], there is an eigensheaf

2010 Mathematics Subject Classification. Primary 14B05, 14C20, 14F18, 32S20.

Key words and phrases. Homogeneous singularity, Log-resolution, Local systems, Multiplier ideals, Finite abelian covers, Hodge spectrum, spectrum multiplicity, Monodromy zeta function.

This research is funded by the Vietnam National Foundation for Science and Technology Development (NAFOSTED) under grant number FWO.101.2015.02.

The first author's research is also supported by ERCEA Consolidator Grant 615655 - NMST and by the Basque Government through the BERC 2014-2017 program and by Spanish Ministry of Economy and Competitiveness MINECO: BCAM Severo Ochoa excellence accreditation SEV-2013-0323. 
decomposition of the $\mathcal{O}_{U}$-module sheaf $\sigma_{*} \mathbb{C}_{M}$ into the unitary local systems $\mathcal{V}_{k}$ on $U$ given by the eigensheaf of $T$ with respect to the eigenvalue $e^{-\frac{2 \pi i k}{d}}, 0 \leq k \leq d-1$, where $\sigma$ is the canonical projection $M \rightarrow U$. On cohomology level, using the Leray spectral sequence, one gets $H^{l}\left(U, \mathcal{V}_{k}\right)$ to be the eigenspace of the monodromy $T$ on $H^{l}(M, \mathbb{C})$ with respect to the eigenvalue $e^{-\frac{2 \pi i k}{d}}$, for any $l$ in $\mathbb{N}$ (cf. [5]). Assume that $D$ has $r$ distinct irreducible components $D_{i}$ and that $m_{i}$ is the multiplicity of $D_{i}$ in $D$. By [5, Lemma 4.2], for each $k$, modulo the identification $R H$ in [4, Theorem 1.2], the local system $\mathcal{V}_{k}$ is nothing but the element $\left(\mathcal{O}_{\mathbb{P} n}\left(\sum_{j=1}^{r}\left\{\frac{k m_{j}}{d}\right\} d_{j}\right),\left(\left\{\frac{k m_{1}}{d}\right\}, \ldots,\left\{\frac{k m_{r}}{d}\right\}\right)\right)$ in the group $\operatorname{Pic}^{\tau}\left(\mathbb{P}^{n}, D\right)$ of realizations of boundaries of $\mathbb{P}^{n}$ on $D$ (cf. [4. Definition 1.1]).

The problem of computing the complex dimension of $H^{l}\left(U, \mathcal{V}_{k}\right)$ can be solved completely under the works by Budur [2, 3, 4, 5] in terms of resolution of singularity. Let $\pi: Y \rightarrow \mathbb{P}^{n}$ be a log-resolution of the family $\left\{D_{1}, \ldots, D_{r}\right\}$, with exceptional divisor $E=\pi^{*}\left(\bigcup_{j=1}^{r} D_{j}\right)=\sum_{j \in A} N_{j} E_{j}, E_{j}$ being irreducible. Denote by $\mathcal{L}^{(k)}$ the invertible sheaf $\pi^{*} \mathcal{O}_{\mathbb{P}^{n}}\left(\sum_{j=1}^{r}\left\{\frac{k m_{j}}{d}\right\} d_{j}\right) \otimes \mathcal{O}_{Y}\left(-\left\lfloor\sum_{j=1}^{r}\left\{\frac{k m_{j}}{d}\right\} \pi^{*} D_{j}\right\rfloor\right)$ on $Y$. As proved in Lemma 3.10, we get

$$
\operatorname{dim}_{\mathbb{C}} H^{l}\left(U, \mathcal{V}_{d-k}\right)=\sum_{p \geq 0} \operatorname{dim}_{\mathbb{C}} H^{l-p}\left(Y, \Omega_{Y}^{p}(\log E) \otimes \mathcal{L}^{(k)^{-1}}\right),
$$

for $l \geq 0$ and $1 \leq k \leq d$, from which the characteristic polynomial $\Delta_{l}(t)$ of $T$ on $H^{l}(M, \mathbb{C})$ follows. Observe that this description is not really useful in practice since it is too difficult to compute the number on the right hand side of the previous equality. However, in the special case where $n=2$ and $l=1$, we obtain in Theorem 4.3 an explicit formula for $\Delta_{1}(t)$ in terms of the multiplier ideal of $\sum_{j=1}^{r}\left\{\frac{k m_{j}}{d}\right\} C_{j}$, where we write $C_{j}$ instead of $D_{j}$ when $D$ is a curve $C$. This is the most important result of the article.

There is another result in the present article, Theorem 4.4, which discusses the relation between the Hodge spectrum and the monodromy zeta function of a homogeneous singularity. In fact, this result can be realized directly from [5. Proposition 4.3] and Proposition 3.8.

\section{Multiplier ideals And Hodge spectrum}

2.1. Multiplier ideals. Let $X$ be a smooth complex algebraic variety and let $D=\left\{D_{1}, \ldots, D_{r}\right\}$ be a family of closed subschemes of $X$. A log-resolution of the family $D$ is a proper birational morphism $\pi: Y \rightarrow X$, where $Y$ is a smooth complex algebraic variety, such that the exceptional set $\operatorname{Ex}(\pi):=$ $\{y \in Y \mid \pi$ is not biregular at $y\}$, the support $\operatorname{Supp}\left(\operatorname{det} \mathrm{Jac}_{\pi}\right)$ of the determinant of the Jacobian of $\pi$, the preimages $\pi^{-1}\left(D_{j}\right), 1 \leq j \leq r$, and the union $\operatorname{Ex}(\pi) \cup \operatorname{Supp}\left(\operatorname{det} \operatorname{Jac}_{\pi}\right) \cup \bigcup_{j=1}^{r} \pi^{-1}\left(D_{j}\right)$ are simple normal crossing divisors. The existence of such a log-resolution is proved by Hironaka. Let $K_{X}$ (resp. $K_{Y}$ ) denote the canonical divisor of $X$ (resp. $Y$ ). Then $K_{Y / X}:=K_{Y}-\pi^{*} K_{X}$ is the divisor defined by det $\mathrm{Jac}_{\pi}$, which is known as the canonical divisor of $\pi$. For any $\alpha=\left(\alpha_{1}, \ldots, \alpha_{r}\right) \in \mathbb{Q}_{>0}^{r}$, we set

$$
\mathcal{J}(X, \alpha D):=\pi_{*} \mathcal{O}_{Y}\left(K_{Y / X}-\left\lfloor\pi^{*}(\alpha D)\right\rfloor\right),
$$

where $\alpha D:=\sum_{j=1}^{r} \alpha_{j} D_{j}$, and $\left\lfloor\pi^{*}(\alpha D)\right\rfloor$ is the round-down of the coefficients of the irreducible components of the divisor $\pi^{*}(\alpha D)$. It is obvious that $\mathcal{J}(X, \alpha D)$ is a sheaf of ideals on $X$, which is an ideal of $\pi_{*} \mathcal{O}_{Y}\left(K_{Y}\right)=\mathcal{O}_{X}$.

Theorem 2.1 (Lazarsfeld [15]). For any $\alpha \in \mathbb{Q}_{>0}^{r}$, the sheaf of ideals $\mathcal{J}(X, \alpha D)$ is independent of the choice of $\pi$, and $R^{i} \mathcal{J}(X, \alpha D)=0$ for $i \geq 1$. The sheaf of ideals $\mathcal{J}(X, \alpha D)$ is called the multiplier ideal of $\alpha D$.

For instance, when $X=\mathbb{C}^{n}$ and $D$ is defined by a monomial ideal $I$, by Howald [13, the multiplier ideal $\mathcal{J}(X, \alpha D)$ is the ideal generated by $x_{1}^{\gamma_{1}} \cdots x_{n}^{\gamma_{n}}$ for all $\left(r_{1}, \ldots, r_{n}\right) \in \mathbb{N}^{n}$ such that $\left(\gamma_{1}+1, \ldots, \gamma_{n}+1\right)$ is in the interior $\operatorname{Int}(\alpha \Gamma(I))$ of $\alpha \Gamma(I)$, where $\Gamma(I)$ is the Newton polyhedron of $I$.

Now let $D$ be a closed subscheme of $X$. A jumping number of $D$ in $X$ is a number $\alpha \in \mathbb{Q}_{>0}$ such that $\mathcal{J}(X, \alpha D) \neq \mathcal{J}(X,(\alpha-\varepsilon) D)$ for all $\varepsilon>0$. The log canonical threshold $\operatorname{lct}(X, D)$ of $(X, D)$ is the smallest jumping number of $D$ in $X$. In 22, Mustata proves a formula of $\operatorname{lct}(X, D)$ in terms of the discrepancies and multiplicities of a $\log$-resolution of $D$. To determine how a singular point affects a 
jumping number, Budur [2] introduces inner jumping multiplicities. By definition, the inner jumping multiplicity $m_{\alpha, \mathbf{p}}(D)$ of $\alpha$ at a closed point $\mathbf{p} \in D$ is the dimension of the complex vector space

$$
\mathcal{K}_{\mathbf{p}}(X, \alpha D):=\mathcal{J}(X,(\alpha-\varepsilon) D) / \mathcal{J}(X,(\alpha-\varepsilon) D+\delta\{\mathbf{p}\}),
$$

for $0<\varepsilon \ll \delta \ll 1$. If $m_{\alpha, \mathbf{p}}(D) \neq 0$, the number $\alpha$ is called an inner jumping number of $(X, D)$ at p. It is proved by Budur in [2, Proposition 2.8] that if $\alpha$ is an inner jumping number of $(X, D)$ at $\mathbf{p}$, for some $\mathbf{p} \in D$, then $\alpha$ is a jumping number of $(X, D)$. Furthermore, Budur can provide an explicit formula computing the number $m_{\alpha, \mathbf{p}}(D)$, which we recall as follows. Let $\pi: Y \rightarrow X$ be a log-resolution of the family $\{D,\{\mathbf{p}\}\}$, with $E=\pi^{*}(D)=\sum_{i \in A} N_{i} E_{i}, E_{i}$ irreducible components, and, for $d \in \mathbb{N}_{>0}$, let $J_{d, \mathbf{p}}:=\left\{i \in A\left|N_{i} \neq 0, d\right| N_{i}, \pi\left(E_{i}\right)=\mathbf{p}\right\}$ and $E_{d, \mathbf{p}}:=\bigcup_{i \in J_{d, \mathbf{p}}} E_{i}$.

Proposition 2.2 (Budur [2], Proposition 2.7). Assume $\alpha=\frac{k}{d}$, with $k$ and $d$ coprime positive integers. Then $m_{\alpha, \mathbf{p}}(D)=\chi\left(Y, \mathcal{O}_{E_{d, \mathbf{p}}}\left(K_{Y / X}-\left\lfloor(1-\varepsilon) \alpha \pi^{*} D\right\rfloor\right)\right)$, where $\chi$ is the sheaf Euler characteristic and $0<\varepsilon \ll 1$.

2.2. Hodge spectrum. Let $X$ be a smooth complex variety of pure dimension $n$, let $f$ be a regular function on $X$ with zero locus $D \neq \emptyset$, and let $\mathbf{p}$ be a closed point in $D_{\text {red }}$. Fixing a smooth metric on $X$ we may define a closed ball $B(\mathbf{p}, \varepsilon)$ around $\mathbf{p}$ in $X$ and a punctured closed disc $D_{\delta}^{*}$ around the origin of $\mathbb{A}_{\mathbb{C}}^{1}$. It is well known (cf. [20]) that, for $0<\delta \ll \varepsilon \ll 1$, the map

$$
f: B(\mathbf{p}, \varepsilon) \cap f^{-1}\left(D_{\delta}^{*}\right) \rightarrow D_{\delta}^{*}
$$

is a smooth locally trivial fibration, called Milnor fibration, whose diffeomorphism type is independent of such $\varepsilon$ and $\delta$. Denote the Milnor fiber $B(\mathbf{p}, \varepsilon) \cap f^{-1}(\delta)$ by $M_{\mathbf{p}}$, the geometric monodromy $M_{\mathbf{p}} \rightarrow M_{\mathbf{p}}$ and its cohomology level $H^{*}\left(M_{\mathbf{p}}, \mathbb{C}\right) \rightarrow H^{*}\left(M_{\mathbf{p}}, \mathbb{C}\right)$ by the same symbol $T$.

Let $\mathrm{MHS}_{\mathbb{C}}^{\text {mon }}$ be the abelian category of complex mixed Hodge structures endowed with an automorphism of finite order. For an object $\left(H, T_{H}\right)$ of $\mathrm{MHS}_{\mathbb{C}}^{\text {mon }}$, one defines its Hodge spectrum as a fractional Laurent polynomial

$$
\operatorname{Hsp}\left(H, T_{H}\right):=\sum_{\alpha \in \mathbb{Q}} n_{\alpha} t^{\alpha},
$$

where $n_{\alpha}:=\operatorname{dim}_{\mathbb{C}} G r_{F}^{\lfloor\alpha\rfloor} H_{e^{2 \pi i \alpha}}, H_{e^{2 \pi i \alpha}}$ is the eigenspace of $T_{H}$ with respect to the eigenvalue $e^{2 \pi i \alpha}$, and $F$ is the Hodge filtration. By [26] and 25], for any $l, H^{l}\left(M_{\mathbf{p}}, \mathbb{C}\right)$ carries a canonical mixed Hodge structure, which is compatible with the semisimple part $T_{s}$ of $T$ so that $\left(H^{l}\left(M_{\mathbf{p}}, \mathbb{C}\right), T_{s}\right)$ is an object of $\mathrm{MHS}_{\mathbb{C}}^{\mathrm{mon}}$. As in [8, Section 4.3] and [2, Section 3], we set

$$
\operatorname{Hsp}^{\prime}(f, \mathbf{p}):=\sum_{j \in \mathbb{Z}}(-1)^{j} \operatorname{Hsp}\left(\widetilde{H}^{n-1+j}\left(M_{\mathbf{p}}, \mathbb{C}\right), T_{s}\right),
$$

where we use the reduced cohomology $\widetilde{H}$ to present the vanishing cycle sheaf cohomology, since $\widetilde{H}^{l}\left(M_{\mathbf{p}}, \mathbb{C}\right)_{e^{2 \pi i \alpha}}=H^{l}\left(M_{\mathbf{p}}, \mathbb{C}\right)_{e^{2 \pi i \alpha}}$ if $l \neq 0$ or $\alpha \notin \mathbb{Z}, \widetilde{H}^{0}\left(M_{\mathbf{p}}, \mathbb{C}\right)_{1}=\operatorname{coker}\left(H^{0}(*, \mathbb{C}) \rightarrow H^{0}\left(M_{\mathbf{p}}, \mathbb{C}\right)_{1}\right)$ (cf. also [6, Section 5.1]). Then the Hodge spectrum of $f$ at $\mathbf{p}$, denoted by $\operatorname{Sp}(f, \mathbf{p})$, is the following

$$
\operatorname{Sp}(f, \mathbf{p})=t^{n} \iota\left(\operatorname{Hsp}^{\prime}(f, \mathbf{p})\right),
$$

where $\iota$ is given by $\iota\left(t^{\alpha}\right)=t^{-\alpha}$. Writing $\operatorname{Sp}(f, \mathbf{p})=\sum_{\alpha \in \mathbb{Q}} n_{\alpha, \mathbf{p}}(f) t^{\alpha}$ one calls the coefficients $n_{\alpha, \mathbf{p}}(f)$ the spectrum multiplicities of $f$ at p. By [6, Proposition 5.2], $n_{\alpha, \mathbf{p}}(f)=0$ if $\alpha$ is a rational number with $\alpha \leq 0$ or $\alpha \geq n$. Moreover, it implies from [5. Corollary 2.3] that, for $\alpha \in(0, n) \cap \mathbb{Q}$,

$$
n_{\alpha, \mathbf{p}}(f)=\sum_{j \in \mathbb{Z}}(-1)^{j} \operatorname{dim}_{\mathbb{C}} G r_{F}^{\lfloor n-\alpha\rfloor} H^{n-1+j}\left(M_{\mathbf{p}}, \mathbb{C}\right)_{e^{-2 \pi i \alpha}}
$$

Specially, using [8, Corollary 4.3.1] and important computations on multiplier ideals, Budur [2] proved the following result, which provides an effective way to compute $n_{\alpha, \mathbf{p}}(f)$, for $\alpha \in(0,1] \cap \mathbb{Q}$.

Theorem 2.3 (Budur [2]). Let $X$ be a smooth quasi-projective complex variety, and $D$ an effective integral divisor on $X$. Assume that $\mathbf{p}$ is a closed point of $D_{\text {red }}$ and $f$ is any local equation of $D$ at $\mathbf{p}$. Then, for any $\alpha \in(0,1] \cap \mathbb{Q}, n_{\alpha, \mathbf{p}}(f)=m_{\alpha, \mathbf{p}}(D)$. 
LÊ, NGUYEN, AND PHO

Remark from Theorem 2.3 that, for $\alpha \in(0,1], t^{\alpha}$ appears in $\operatorname{Sp}(f, \mathbf{p})$ if and only if $\alpha$ is an inner jumping number of $(X, D)$ at $\mathbf{p}$. If $\mathbf{p}$ is an isolated singularity of $D$, Theorem 2.3 may be even applied to the previous remark when replacing $X$ by an open neighborhood of $X$ to obtain Varchenko's result [28] (see Corollary in [2, Section 1]).

\section{LOCAL Systems And Milnor fiBers of homegeneous Singularities}

3.1. Local systems and normal $G$-covers. Let us recall some basic notions of local systems and cyclic covers in [12 and [4. A complex local system $\mathcal{V}$ on a complex manifold is a locally constant sheaf of finite dimensional complex vector spaces. The rank of a locally constant sheaf is the dimension of a stalk as a complex vector space. As mentioned in Budur [4, local systems of rank one on a complex manifold $U$ correspond to morphisms of groups $H_{1}(U) \rightarrow \mathbb{C}^{*}$. In this correspondence, a local system is called unitary if it is sent to a morphism of groups $H_{1}(U) \rightarrow S^{1}=\left\{\eta \in \mathbb{C}^{*}|| \eta \mid=1\right\}$. The constant sheaf $\mathbb{C}_{U}$ and any local system of rank one of finite order are simple examples of unitary local systems.

Let $X$ be a smooth complex projective variety of dimension $n$, and $f$ a regular function on $X$ with zero divisor $D:=f^{-1}(0)$. Denote $U:=X \backslash D$ and write $D_{\text {red }}=\bigcup_{j=1}^{r} D_{j}$, where $D_{j}$ are distinct irreducible reduced subvarieties of $D$. We may use $D$ as the family $\left\{D_{1}, \ldots, D_{r}\right\}$ by abuse of notation (and the following definition will be in this sense), we write $c_{1}(\mathcal{L})$ for the first Chern class of a line bundle $\mathcal{L}$ and consider the group

$$
\operatorname{Pic}^{\tau}(X, D):=\left\{(\mathcal{L}, \alpha) \in \operatorname{Pic}(X) \times[0,1)^{r} \mid c_{1}(\mathcal{L})=\alpha[D] \in H^{2}(X, \mathbb{R})\right\} .
$$

with the following operation

$$
\left.(\mathcal{L}, \alpha) \cdot\left(\mathcal{L}^{\prime}, \alpha^{\prime}\right):=\left(\mathcal{L} \otimes \mathcal{L}^{\prime} \otimes \mathcal{O}_{X}\left(-\left\lfloor\left(\alpha+\alpha^{\prime}\right) D\right\rfloor\right)\right),\left\{\alpha+\alpha^{\prime}\right\}\right),
$$

where $\alpha[D]:=\sum_{j=1}^{r} \alpha_{j}\left[D_{j}\right]$, an $\mathbb{R}$-linear combination of the cohomology classes $\left[D_{j}\right]$ in $H^{2}(X, \mathbb{R})$, and as above $\alpha D:=\sum_{j=1}^{r} \alpha_{j} D_{j},\lfloor\alpha\rfloor:=\left(\left\lfloor\alpha_{1}\right\rfloor, \ldots,\left\lfloor\alpha_{r}\right\rfloor\right)$ and $\{\alpha\}:=\alpha-\lfloor\alpha\rfloor$.

Theorem 3.1 (Budur 4, Theorem 1.2). There is a canonical isomorphism of groups

$$
R H: \operatorname{Pic}^{\tau}(X, D) \cong \operatorname{Hom}\left(H_{1}(U), S^{1}\right),
$$

By this, one may identify a unitary local system of rank one on $U$ with an element of $\operatorname{Pic}^{\tau}(X, D)$.

Let $\pi: Y \rightarrow X$ be a log-resolution of the family $\left\{D_{1}, \ldots, D_{r}\right\}$, and $E:=Y \backslash \pi^{-1}(U)=\sum_{i \in A} N_{i} E_{i}$, with $E_{j}$ irreducible. We shall use the following two important results.

Proposition 3.2 (Budur [4, Proposition 3.3). The map $\pi_{p a r}^{*}: \operatorname{Pic}^{\tau}(X, D) \rightarrow \operatorname{Pic}^{\tau}(Y, E)$ which sends $(\mathcal{L}, \alpha)$ to $\left(\pi^{*} \mathcal{L}-\lfloor\beta E\rfloor,\{\beta\}\right)$ with $\beta$ defined by $\pi^{*}(\alpha D)=\beta E$ is an isomorphism of groups.

Theorem 3.3 (Budur 3], Theorem 4.6). Let $\mathcal{V}$ be a rank one unitary local system on $U$ which corresponds to $(\mathcal{L}, \alpha) \in \operatorname{Pic}^{\tau}(X, D)$. Then, for all $p, q \in \mathbb{N}$, we have

$$
G r_{F}^{p} H^{p+q}\left(U, \mathcal{V}^{\vee}\right)=H^{n-q}\left(Y, \Omega_{Y}^{p}(\log E)^{\vee} \otimes \omega_{Y} \otimes \pi^{*} \mathcal{L} \otimes \mathcal{O}_{Y}\left(-\left\lfloor\pi^{*}(\alpha D)\right\rfloor\right)\right)^{\vee} .
$$

In particular,

$$
G r_{F}^{0} H^{q}\left(U, \mathcal{V}^{\vee}\right)=H^{n-q}\left(X, \omega_{X} \otimes \mathcal{L} \otimes \mathcal{J}(X, \alpha D)\right)^{\vee} .
$$

Let $G$ be a finite group. By [4, Corollary 1.10], the dual group $G^{*}=\operatorname{Hom}\left(G, \mathbb{C}^{*}\right)$ of $G$ gives rise to a normal $G$-cover of $X$ unramified above $U$. Namely, the normal $G$-cover of $X$ is the morphism

$$
\phi: \widetilde{X}=\operatorname{Spec}_{\mathcal{O}_{X}}\left(\bigoplus_{\eta \in G^{*}} \mathcal{L}_{\eta}^{-1}\right) \rightarrow X
$$

induced by the $\mathcal{O}_{X}$-module structural morphisms $\mathcal{O}_{X} \rightarrow \mathcal{L}_{\eta}$, for all $\eta \in G^{*}$, where we identify $G^{*}$ with the subgroup $\left\{\left(L_{\eta}, \alpha_{\eta}\right) \mid \eta \in G^{*}\right\}$ of $\operatorname{Pic}^{\tau}(X, D)$. The group $G$ acts on $\mathcal{L}_{\eta}^{-1}$ via the character $\eta$, hence acts on the $\mathcal{O}_{X}$-module sheaf $\phi_{*} \mathcal{O}_{\tilde{X}}$. By [4, Corollary 1.11], $\phi_{*} \mathcal{O}_{\widetilde{X}}$ admits an eigensheaf decomposition

$$
\phi_{*} \mathcal{O}_{\widetilde{X}}=\bigoplus_{\eta \in G^{*}} \mathcal{L}_{\eta}^{-1},
$$


where the eigensheaf $\mathcal{L}_{\eta}^{-1}$ is with respect to the eigenvalue $\eta$ of the action of $G$ on $\phi_{*} \mathcal{O}_{\tilde{X}}$.

Now we consider the log-resolution $\pi$. By Proposition [3.2. since $\left\{\left(\mathcal{L}_{\eta}, \alpha_{\eta}\right) \mid \eta \in G^{*}\right\}$ is a finite subgroup of $\operatorname{Pic}^{\tau}(X, D),\left\{\left(\pi^{*} \mathcal{L}_{\eta}-\left\lfloor\beta_{\eta} E\right\rfloor, \beta_{\eta}\right) \mid \eta \in G^{*}\right\}$, with $\beta_{\eta}$ defined by $\pi^{*}\left(\alpha_{\eta} D\right)=\beta_{\eta} E$, is a finite subgroup of $\operatorname{Pic}^{\tau}(Y, E)$. By the same way as previous we can construct the corresponding normal $G$-cover of $Y$ unramified above $\pi^{-1}(U) \cong U$ as follows

$$
\rho: \tilde{Y}=\operatorname{Spec}_{\mathcal{O}_{Y}}\left(\bigoplus_{\eta \in G^{*}} \pi^{*} \mathcal{L}_{\eta}^{-1} \otimes \mathcal{O}_{Y}\left(\left\lfloor\beta_{\eta} E\right\rfloor\right)\right) \rightarrow Y,
$$

where the group $G$ of acts on $\widetilde{Y}$ and on $\rho_{*} \mathcal{O}_{\widetilde{Y}}$. Moreover, similarly as (3.2), we have

Proposition 3.4 (Budur 4, Corollary 1.12). There is an eigensheaf decomposition

$$
\rho_{*} \mathcal{O}_{\widetilde{Y}}=\bigoplus_{\eta \in G^{*}} \pi^{*} \mathcal{L}_{\eta}^{-1} \otimes \mathcal{O}_{Y}\left(\left\lfloor\beta_{\eta} E\right\rfloor\right),
$$

the eigensheaf $\pi^{*} \mathcal{L}_{\eta}^{-1} \otimes \mathcal{O}_{Y}\left(\left\lfloor\beta_{\eta} E\right\rfloor\right)$ is with respect to the eigenvalue $\eta$ of the action of $G$ on $\rho_{*} \mathcal{O}_{\widetilde{Y}}$.

3.2. Milnor fibers of homegeneous singularity. Let $f\left(x_{0}, \ldots, x_{n}\right) \in \mathbb{C}\left[x_{0}, \ldots, x_{n}\right]$ be a homogeneous polynomial of degree $d$. We shall take $f$ into two closely interactive entities, a Milnor fiber at the origin of $\mathbb{C}^{n+1}$ and a complex projective hypersurface of $\mathbb{P}^{n}$. By [20, Lemma 9.4], the Minor fiber $M$ of $f$ at the origin of $\mathbb{C}^{n+1}$ is diffeomorphic to $\left\{\left(x_{0}, \ldots, x_{n}\right) \in \mathbb{C}^{n+1} \mid f\left(x_{0}, \ldots, x_{n}\right)=1\right\}$. The geometric monodromy $M \rightarrow M$ is given by multiplication of elements of $M$ by $e^{\frac{2 \pi i}{d}}$, which induces an endomorphism $T$ of the complex vector space $H^{*}(M, \mathbb{C})$.

Following [5, Section 4], we consider the smooth complex projective variety $X=\mathbb{P}^{n}$ and the closed subscheme $D$ of $X$ defined by the zero locus of $f$. Put $U:=X \backslash D$. Since the action of $\mathbb{Z} / d \mathbb{Z}$ on $M$ is free, we have a natural isomorphism $M /(\mathbb{Z} / d \mathbb{Z}) \cong U$. Denote by $\sigma$ the quotient map $M \rightarrow U$, which is the cyclic cover of degree $d$ of $U$. Then there is an eigensheaf decomposition of the $\mathcal{O}_{U}$-module sheaf $\sigma_{*} \mathbb{C}_{M}$ as follows

$$
\sigma_{*} \mathbb{C}_{M}=\bigoplus_{k=0}^{d-1} \mathcal{V}_{k}
$$

where $\mathcal{V}_{k}$ is the unitary local system on $U$ given by the eigensheaf of $T$ with respect to the eigenvalue $e^{-\frac{2 \pi i k}{d}}$. This implies that

$$
H^{l}\left(U, \sigma_{*} \mathbb{C}_{M}\right)=\bigoplus_{k=0}^{d-1} H^{l}\left(U, \mathcal{V}_{k}\right)
$$

Let us consider the Leray spectral sequence

$$
E_{2}^{p, q}=H^{q}\left(U, R^{p} \sigma_{*} \mathbb{C}_{M}\right) \Rightarrow H^{p+q}\left(M, \mathbb{C}_{M}\right) .
$$

Since $\sigma$ is a finite morphism of schemes, $R^{p} \sigma_{*} \mathbb{C}_{M}=0$ for all $p \geq 1$, hence, by this spectral sequence, we have $H^{l}\left(U, \sigma_{*} \mathbb{C}_{M}\right)=H^{l}\left(M, \mathbb{C}_{M}\right)=H^{l}(M, \mathbb{C})$, for $l \in \mathbb{N}$. This implies the following important lemma (cf. [5, Section 4]).

Lemma 3.5 (Budur [5]). The complex vector space $H^{l}\left(U, \mathcal{V}_{k}\right)$ if nontrivial is the eigenspace of the monodromy action $T$ on $H^{l}(M, \mathbb{C})$ with respect to the eigenvalue $e^{-\frac{2 \pi i k}{d}}$, that is,

$$
H^{l}(M, \mathbb{C})_{e^{-\frac{2 \pi i k}{d}}}=H^{l}\left(U, \mathcal{V}_{k}\right)
$$

for $0 \leq k \leq d-1$ and $l \geq 0$.

In fact, there are two commuting monodromy actions on $H^{l}(M, \mathbb{C})$, where the endomorphism $T$ is the first one. The second one is, for each $k$, the monodromy of $\mathcal{V}_{k}$ around a generic point of $D_{j}$, which, by [5. Lemma 4.1], is given by multiplication by $e^{\frac{2 \pi i k m_{j}}{d}}$. Together with [4, Proposition 3.3], it proves the following important lemma. 
Lemma 3.6 (Budur [5], Lemma 4.2). Assume $D=\sum_{j=1}^{r} m_{j} D_{j}$, with $D_{j}$ irreducible of degree $d_{j}$. Then the element in $\operatorname{Pic}^{\tau}(X, D)$ corresponding via the isomorphism RH in Theorem 3.1 to the unitary local system $\mathcal{V}_{k}$ is $\left(\mathcal{O}_{\mathbb{P} n}\left(\sum_{j=1}^{r}\left\{\frac{k m_{j}}{d}\right\} d_{j}\right),\left(\left\{\frac{k m_{1}}{d}\right\}, \ldots,\left\{\frac{k m_{r}}{d}\right\}\right)\right)$.

Notice that $\sum_{j=1}^{r}\left\{\frac{k m_{j}}{d}\right\} d_{j}$ is an integer, because, if for every $1 \leq j \leq r$ we write $k m_{j}=d n_{j}+s_{j}$, with $n_{j}, s_{j} \in \mathbb{N}, 0 \leq s_{j}<d$, we have

$$
\sum_{j=1}^{r}\left\{\frac{k m_{j}}{d}\right\} d_{j}=\sum_{j=1}^{r} \frac{s_{j} d_{j}}{d}=\sum_{j=1}^{r} \frac{k m_{j} d_{j}-d n_{j} d_{j}}{d}=k-\sum_{j=1}^{r} n_{j} d_{j} .
$$

Fix a log-resolution $\pi: Y \rightarrow \mathbb{P}^{n}$ of the family of closed subschemes $\left\{D_{1}, \ldots, D_{r}\right\}$ of $\mathbb{P}^{n}$, and, as previous, denote $E=\pi^{*}\left(\bigcup_{j=1}^{r} D_{j}\right)=\sum_{j \in A} N_{j} E_{j}$, with $E_{j}$ irreducible components of $\pi^{-1}(D)$. Let

$$
\mathcal{L}^{(k)}:=\pi^{*} \mathcal{O}_{\mathbb{P}^{n}}\left(\sum_{j=1}^{r}\left\{\frac{k m_{j}}{d}\right\} d_{j}\right) \otimes \mathcal{O}_{Y}\left(-\left\lfloor\sum_{j=1}^{r}\left\{\frac{k m_{j}}{d}\right\} \pi^{*} D_{j}\right\rfloor\right) .
$$

Denote by $B$ the set of integers $k$ such that $0 \leq k \leq d-1$ and $d$ divides $k m_{j}$ for all $1 \leq j \leq r$, and by $\bar{B}$ the complement of $B$ in $[0, d-1] \cap \mathbb{Z}$.

Remark 3.7. If $k$ is in $B$, then $\mathcal{L}^{(k)}=\mathcal{O}_{Y}$. Furthermore, if $k$ is in $B$ and $k \neq 0$, so is $d-k$; if $k$ and $k^{\prime}$ are in $B$, so is either $k+k^{\prime}$ or $k+k^{\prime}-d$; hence we can consider $B$ as a subgroup of $\mathbb{Z} / d \mathbb{Z}$. Let $m=\operatorname{gcd}\left(m_{1}, \ldots, m_{r}\right)$, and $u_{j} \in \mathbb{N}_{>0}$ with $m_{j}=m u_{j}$ for $1 \leq j \leq r$. Then $k \in B$ if and only if $0 \leq k \leq d-1$ and $k u_{s}$ is divisible by $\sum_{j=1}^{r} d_{j} u_{j}$ for any $1 \leq s \leq r$. Since $u_{1}, \ldots, u_{r}$ are coprime, the latter means that $k$ is divisible by $\sum_{j=1}^{r} d_{j} u_{j}$, hence the cardinal $|B|$ of $B$ equals $m$.

For simplicity of notation, from now on, if $\mathcal{A}$ is a sheaf on $\mathbb{P}^{n}$, and $l \in \mathbb{Z}$, we shall write $\mathcal{A}(l)$ in stead of $\mathcal{A} \otimes \mathcal{O}_{\mathbb{P}^{n}}(l)$.

Proposition 3.8. With the notation as in Lemma 3.6 we have

(i) $\operatorname{dim}_{\mathbb{C}} G r_{F}^{p} H^{p+q}\left(U, \mathcal{V}_{k}\right)=\operatorname{dim}_{\mathbb{C}} H^{q}\left(Y, \Omega_{Y}^{p}(\log E)\right)$, for $k \in B$;

(ii) $\operatorname{dim}_{\mathbb{C}} G r_{F}^{p} H^{p+q}\left(U, \mathcal{V}_{d-k}\right)=\operatorname{dim}_{\mathbb{C}} H^{q}\left(Y, \Omega_{Y}^{p}(\log E) \otimes \mathcal{L}^{(k)^{-1}}\right)$, for $k \in \bar{B}$.

In particular, for $k \in \bar{B}$,

$$
\operatorname{dim}_{\mathbb{C}} G r_{F}^{0} H^{q}\left(U, \mathcal{V}_{d-k}\right)=\operatorname{dim}_{\mathbb{C}} H^{n-q}\left(\mathbb{P}^{n}, \mathcal{J}\left(\mathbb{P}^{n}, \sum_{j=1}^{r}\left\{\frac{k m_{j}}{d}\right\} D_{j}\right)\left(\sum_{j=1}^{r}\left\{\frac{k m_{j}}{d}\right\} d_{j}-n-1\right)\right) .
$$

Proof. Due to the group law (3.1) of $\operatorname{Pic}^{\tau}(X, D)$ and definition of $\mathcal{V}_{k}$, it is obvious that $\mathcal{V}_{k}=\mathcal{V}_{k}^{\vee}=\mathcal{V}_{0}$ for $k \in B$, and that $\mathcal{V}_{d-k}=\mathcal{V}_{k}^{\vee}$ for $k \in \bar{B}$. Then, by Lemma 3.6 and Theorem 3.3, we have

$$
G r_{F}^{p} H^{p+q}\left(U, \mathcal{V}_{k}\right)=H^{n-q}\left(Y, \Omega_{Y}^{p}(\log E)^{\vee} \otimes \omega_{Y}\right)^{\vee}
$$

for $k \in B$, and

$$
\begin{aligned}
G r_{F}^{p} H^{p+q}\left(U, \mathcal{V}_{d-k}\right) & =H^{n-q}\left(Y, \Omega_{Y}^{p}(\log E)^{\vee} \otimes \omega_{Y} \otimes \mathcal{L}^{(k)}\right)^{\vee} \\
& =H^{n-q}\left(Y,\left(\Omega_{Y}^{p}(\log E) \otimes \mathcal{L}^{(k)^{-1}}\right)^{\vee} \otimes \omega_{Y}\right)^{\vee},
\end{aligned}
$$

for $k \in \bar{B}$. Applying the Serre duality we obtain (i) and (ii).

For the rest statement, we again apply Lemma 3.6 and the particular case in Theorem 3.3 , together with the definition of multiplier ideal.

Denote $\mathcal{L}_{\text {red }}^{(k)}:=\pi^{*} \mathcal{O}_{\mathbb{P}^{n}}(k) \otimes \mathcal{O}_{Y}\left(-\left\lfloor\frac{k}{d} E\right\rfloor\right)$, for $0 \leq k \leq d-1$.

Corollary 3.9. With the notation as in Lemma 3.6 and $D$ being reduced, for $1 \leq k \leq d$,

(i) $\operatorname{dim}_{\mathbb{C}} G r_{F}^{p} H^{p+q}\left(U, \mathcal{V}_{d-k}\right)=\operatorname{dim}_{\mathbb{C}} H^{q}\left(Y, \Omega_{Y}^{p}(\log E) \otimes \mathcal{L}_{\text {red }}^{(k)}{ }^{-1}\right)$;

(ii) $\operatorname{dim}_{\mathbb{C}} G r_{F}^{0} H^{q}\left(U, \mathcal{V}_{d-k}\right)=\operatorname{dim}_{\mathbb{C}} H^{n-q}\left(\mathbb{P}^{n}, \mathcal{J}\left(\mathbb{P}^{n}, \frac{k}{d} D\right)(k-n-1)\right)$.

Proof. Applying Proposition 3.8 to the special case $m_{1}=\cdots=m_{r}=1$ we obtain the statements. Note that, in this case, $B=\{0\}$ and $\bar{B}=\{1, \ldots, d-1\}$. 
Lemma 3.10. With the notation as in Lemma 3.6, and by observation $\mathcal{L}^{(d)}=\mathcal{L}^{(0)}$, we have

(i) $\operatorname{dim}_{\mathbb{C}} H^{1}\left(U, \mathcal{V}_{k}\right)=r-1$, if $n=2$ and $k \in B$;

(ii) $\operatorname{dim}_{\mathbb{C}} H^{l}\left(U, \mathcal{V}_{d-k}\right)=\sum_{p \geq 0} \operatorname{dim}_{\mathbb{C}} H^{j-p}\left(Y, \Omega_{Y}^{p}(\log E) \otimes \mathcal{L}^{(k)^{-1}}\right)$, if $j \geq 0$ and $1 \leq k \leq d$.

Proof. By Proposition 3.8 (i), if $k \in B$, we have $\operatorname{dim}_{\mathbb{C}} G r_{F}^{p} H^{p+q}\left(U, \mathcal{V}_{k}\right)=\operatorname{dim}_{\mathbb{C}} H^{q}\left(Y, \Omega_{Y}^{p}(\log E)\right)$, thus

$$
\operatorname{dim}_{\mathbb{C}} H^{1}\left(U, \mathcal{V}_{0}\right)=\operatorname{dim}_{\mathbb{C}} H^{1}\left(Y, \mathcal{O}_{Y}\right)+\operatorname{dim}_{\mathbb{C}} H^{0}\left(Y, \Omega_{Y}^{1}(\log E)\right) .
$$

Assume that $n=2$. It is a fact that $\operatorname{dim}_{\mathbb{C}} H^{1}\left(Y, \mathcal{O}_{Y}\right)=0$, because $Y$ is birationally equivalent to $\mathbb{P}^{2}$, and that $\operatorname{dim}_{\mathbb{C}} H^{0}\left(Y, \Omega_{Y}^{1}(\log E)\right)=r-1$, due to the proof of Théorème 6 in [11. This proves (i).

The statement (ii) of this lemma is a consequence of Proposition 3.8 (ii).

\section{Monodromy Characteristic POlynomials AND ZETA FUnCtion}

As in Section 3.2 we shall work with a homogeneous polynomial $f\left(x_{0}, \ldots, x_{n}\right) \in \mathbb{C}\left[x_{0}, \ldots, x_{n}\right]$ of degree $d$. By considering its germ at the origin of $\mathbb{C}^{n+1}$ we study some singularity invariants, including the characteristic polynomials and the zeta function of the monodromy.

4.1. Characteristic polynomials. Recall that the Milnor fiber $M$ of the singularity $f\left(x_{0}, \ldots, x_{n}\right)$ at the origin of $\mathbb{C}^{n+1}$ is diffeomorphic to $\left\{\left(x_{0}, \ldots, x_{n}\right) \in \mathbb{C}^{n+1} \mid f\left(x_{0}, \ldots, x_{n}\right)=1\right\}$, and the monodromy $T$ is induced by $e^{\frac{2 \pi i}{d}} \cdot\left(x_{0}, \ldots, x_{n}\right)=\left(e^{\frac{2 \pi i}{d}} x_{0}, \ldots, e^{\frac{2 \pi i}{d}} x_{n}\right)$. By definition, the (monodromy) characteristic polynomial $\Delta_{l}(t)$ of the endomorphism $\left.T\right|_{H^{l}(M, \mathbb{C})}$ of $H^{l}(M, \mathbb{C})$ is the monic polynomial

$$
\Delta_{l}(t)=\operatorname{det}\left(t \operatorname{Id}-\left.T\right|_{H^{l}(M, \mathbb{C})}\right) .
$$

Assume that

$$
f\left(x_{0}, \ldots, x_{n}\right)=\prod_{j=1}^{r} f_{j}\left(x_{0}, \ldots, x_{n}\right)^{m_{j}},
$$

where $f_{j}\left(x_{0}, \ldots, x_{n}\right)$ are distinct irreducible homogeneous polynomials of degree $d_{j}, 1 \leq j \leq r$. As above, we denote by $D_{j}$ the complex projective plane curve $\left\{\left(x_{0}: \cdots: x_{n}\right) \in \mathbb{P}^{n} \mid f_{j}\left(x_{0}, \ldots, x_{n}\right)=0\right\}$, for $1 \leq j \leq r$.

Fix a log-resolution $\pi: Y \rightarrow \mathbb{P}^{n}$ of the family $D=\left\{D_{1}, \ldots, D_{r}\right\}$, with normal crossing divisor $E=\pi^{-1}\left(\bigcup_{j=1}^{r} D_{j}\right)$. As mentioned in Section 3] there is an isomorphism $M /(\mathbb{Z} / d \mathbb{Z}) \cong U=\mathbb{P}^{n} \backslash D$ so that the canonical projection $\sigma: M \rightarrow U$ induces an eigensheaf decomposition $\sigma_{*} \mathbb{C}_{M}=\bigoplus_{k=0}^{d-1} \mathcal{V}_{k}$, where $\mathcal{V}_{k}$ are the unitary local systems on $U$ given in Lemma 3.6. By Lemma 3.5. for $1 \leq k \leq d$ and $l \in \mathbb{N}$, the vector space $H^{l}\left(U, \mathcal{V}_{d-k}\right)$ if nontrivial is the eigenspace of $\left.T\right|_{H^{l}(M, \mathbb{C})}$ with respect to the eigenvalue $e^{\frac{2 \pi i k}{d}}$. This together with Lemma 3.10 and Remark 3.7 proves the following lemma.

Lemma 4.1. Let $\Delta_{l}(t)$ be the characteristic polynomial of the endomorphism $\left.T\right|_{H^{l}(M, \mathbb{C})}$ of $H^{l}(M, \mathbb{C})$. Then, with the previous notation and $l \in \mathbb{N}$, one has

$$
\triangle_{l}(t)=\prod_{k=0}^{d-1}\left(t-e^{\frac{2 \pi i k}{d}}\right)^{h_{l}^{(k)}},
$$

where,

$$
h_{l}^{(k)}:=\operatorname{dim}_{\mathbb{C}} H^{l}\left(U, \mathcal{V}_{d-k}\right)=\sum_{p+q=l} h^{q}\left(\Omega_{Y}^{p}(\log E) \otimes \mathcal{L}^{(k)^{-1}}\right),
$$

with $h^{q}\left(\Omega_{Y}^{p}(\log E) \otimes \mathcal{L}^{(k)^{-1}}\right)=\operatorname{dim}_{\mathbb{C}} H^{q}\left(Y, \Omega_{Y}^{p}(\log E) \otimes \mathcal{L}^{(k)^{-1}}\right)$, and

$$
\mathcal{L}^{(k)}=\pi^{*} \mathcal{O}_{\mathbb{P}^{n}}\left(\sum_{j=1}^{r}\left\{\frac{k m_{j}}{d}\right\} d_{j}\right) \otimes \mathcal{O}_{Y}\left(-\left\lfloor\sum_{j=1}^{r}\left\{\frac{k m_{j}}{d}\right\} \pi^{*} D_{j}\right\rfloor\right) .
$$

As above, we denote by $B$ the set of $k$ in $\mathbb{Z}$ such that $0 \leq k \leq d-1$ and $d$ divides $k m_{j}$ for all $1 \leq j \leq r$, by $\bar{B}$ the complement of $B$ in $[0, d-1] \cap \mathbb{Z}$, and $m=\operatorname{gcd}\left(m_{1}, \ldots, m_{r}\right)$. Due to Remark 3.7 , $B$ may be considered as a subgroup of $\mathbb{Z} / d \mathbb{Z}$. Let $G$ be the quotient group $(\mathbb{Z} / d \mathbb{Z}) / B$. For convenience, we shall identify $k \in[0, d-1] \cap \mathbb{Z}$ with its class in $G$. 
Lemma 4.2. With the notation as in Lemma 4.1, one has

$$
\Delta_{l}(t)=\prod_{k \in G}\left(t^{m}-e^{\frac{2 \pi i k m}{d}}\right)^{h_{l}^{(k)}}
$$

for $l \in \mathbb{N}$. In particular, $\Delta_{0}(t)=t^{m}-1$.

Proof. If $k$ and $k^{\prime}$ belong to the same class in $G$, we have $h_{l}^{(k)}=h_{l}^{\left(k^{\prime}\right)}$. This together with Lemma 4.1 implies the first statement. Since $h^{0}\left(\mathcal{O}_{Y}\right)=1$, it remains to check that $h^{0}\left(\mathcal{L}^{(k)^{-1}}\right)=0$ for $k \in G \backslash\{0\}$. By Lemmas 3.5 and 3.10 , we have

$$
\operatorname{dim}_{\mathbb{C}} H^{0}(M, \mathbb{C})=\sum_{k \in B} h^{0}\left(\mathcal{L}^{(k)^{-1}}\right)+\sum_{k \in \bar{B}} h^{0}\left(\mathcal{L}^{(k)^{-1}}\right) .
$$

It is known that $\operatorname{dim}_{\mathbb{C}} H^{0}(M, \mathbb{C})=m$ (cf. [9, Proposition 2.3]). Note that $|B|=m$ (cf. Remark 3.7), and that, for $k \in B, \mathcal{L}^{(k)}=\mathcal{O}_{Y}$ and $h^{0}\left(\mathcal{O}_{Y}\right)=1$. Then (4.1) is equivalent to $\sum_{k \in \bar{B}} h^{0}\left(\mathcal{L}^{(k)^{-1}}\right)=0$, which implies that $h^{0}\left(\mathcal{L}^{(k)^{-1}}\right)=0$ for $k \in \bar{B}$; in particular, $h^{0}\left(\mathcal{L}^{(k)^{-1}}\right)=0$ for $k \in G \backslash\{0\}$.

Let us now consider the case where $n=2$. In this case, we shall denote $C$ (resp. $\left.C_{j}\right)$ instead of $D$ (resp. $D_{j}$ ). Then the characteristic polynomial $\Delta_{1}(t)$ is an important invariant of the homogeneous surface singularity. The following theorem is a main result in the present article.

Theorem 4.3. With the previous notation and $n=2$, one has

$$
\Delta_{1}(t)=\left(t^{m}-1\right)^{r-1} \prod_{k \in G \backslash\{0\}}\left(t^{2 m}-2 t^{m} \cos \frac{2 k m \pi}{d}+1\right)^{\ell_{k}},
$$

where

$$
\ell_{k}:=\operatorname{dim}_{\mathbb{C}} H^{1}\left(\mathbb{P}^{2}, \mathcal{J}\left(\mathbb{P}^{2}, \sum_{j=1}^{r}\left\{\frac{k m_{j}}{d}\right\} C_{j}\right)\left(\sum_{j=1}^{r}\left\{\frac{k m_{j}}{d}\right\} d_{j}-3\right)\right) .
$$

Proof. According to Lemma 4.2, it suffices to prove that

$$
h^{1}\left(\mathcal{L}^{(k)^{-1}}\right)=\ell_{k}
$$

and

$$
h^{0}\left(\Omega_{Y}^{1}(\log E) \otimes \mathcal{L}^{(k)^{-1}}\right)=\ell_{d-k},
$$

for $k \in G \backslash\{0\}$. The equality (4.2) is a direct corollary of Proposition 3.8 and Lemma 3.10

To prove (4.3) we consider a common $G$-equivariant desingularization of $\widetilde{X}$ and $\widetilde{Y}$, say, $\theta: Z \rightarrow \widetilde{X}$ and $\nu: Z \rightarrow \widetilde{Y}$, in the sense of [1], such that $\pi \circ \rho \circ \nu=\phi \circ \theta=: u$. Here, we use the notation in Section 3.1 with $X=\mathbb{P}^{2}$, and, in particular, we use the the normal $G$-cover of $\mathbb{P}^{2}$,

$$
\phi: \widetilde{X}=\operatorname{Spec}_{\mathcal{O}_{\mathbb{P}^{2}}}\left(\bigoplus_{k \in G} \mathcal{O}_{\mathbb{P}^{2}}\left(-\sum_{j=1}^{r}\left\{\frac{k m_{j}}{d}\right\} d_{j}\right)\right) \rightarrow \mathbb{P}^{2},
$$

and the normal $G$-cover of $Y$,

$$
\rho: \widetilde{Y}=\operatorname{Spec}_{\mathcal{O}_{Y}}\left(\bigoplus_{k \in G} \mathcal{L}^{(k)^{-1}}\right) \rightarrow Y
$$

where as mentioned previously we identify $k \in[0, d-1] \cap \mathbb{Z}$ with its class in $G$.

Note that $G^{*}=\left\{\left(\mathcal{O}_{\mathbb{P}^{2}}\left(\sum_{j=1}^{r}\left\{\frac{k m_{j}}{d}\right\} d_{j}\right),\left(\left\{\frac{k m_{1}}{d}\right\}, \ldots,\left\{\frac{k m_{r}}{d}\right\}\right)\right)\right\}_{0 \leq k \leq d-1}$, which is by Remark 3.7 a subgroup of order $\frac{d}{m}$ of the group $\operatorname{Pic}^{\tau}\left(\mathbb{P}^{2}, C\right)$. We may choose $Z$ such that $\Delta:=Z \backslash u^{-1}(U)$ is a normal crossing divisor. An analogue of [11, Corollaire 4] shows that, for any $q \in \mathbb{N}$,

$$
\begin{aligned}
(\rho \circ \nu)_{*} \Omega_{Z}^{q}(\log \Delta) & \cong \Omega_{Y}^{q}(\log E) \otimes(\rho \circ \nu)_{*} \mathcal{O}_{Z}, \\
R^{p}(\rho \circ \nu)_{*} \Omega_{Z}^{q}(\log \Delta) & =0 \quad \text { if } p>0
\end{aligned}
$$


(see also [12, Lemma 3.22]). By the Leray spectral sequence

$$
E_{2}^{p, q}=H^{q}\left(Y, R^{p}(\rho \circ \nu)_{*} \Omega_{Z}^{1}(\log \Delta)\right) \Rightarrow H^{p+q}\left(Z, \Omega_{Z}^{1}(\log \Delta)\right)
$$

and by (4.4), we get, in particular,

$$
H^{0}\left(Y, \Omega_{Y}^{1}(\log E) \otimes(\rho \circ \nu)_{*} \mathcal{O}_{Z}\right)=H^{0}\left(Z, \Omega_{Z}^{1}(\log \Delta)\right) .
$$

Since $G^{*}$ is a finite subgroup of $\operatorname{Pic}^{\tau}\left(\mathbb{P}^{2}, C\right)$, we deduce from Proposition 3.4 that

$$
(\rho \circ \nu)_{*} \mathcal{O}_{Z}=\rho_{*} \mathcal{O}_{\widetilde{Y}}=\bigoplus_{k \in G} \mathcal{L}^{(k)^{-1}}
$$

This yields the following decomposition

$$
H^{0}\left(Y, \Omega_{Y}^{1}(\log E) \otimes(\rho \circ \nu)_{*} \mathcal{O}_{Z}\right)=\bigoplus_{k \in G} H^{0}\left(Y, \Omega_{Y}^{1}(\log E) \otimes \mathcal{L}^{(k)^{-1}}\right) .
$$

Note that, due to the proof of Lemma 3.10, the direct summand of (4.6) corresponding to $k=0$ has complex dimension $r-1$.

Now we compute the dimension of complex vector space on the right hand side of (4.5). Similarly as in the proof of Lemma 7 of [11, one may point out that

$$
\operatorname{dim}_{\mathbb{C}} H^{0}\left(Z, \Omega_{Z}^{1}(\log \Delta)\right)=\operatorname{dim}_{\mathbb{C}} H^{0}\left(Z, \Omega_{Z}^{1}\right)+(r-1) .
$$

On the other hand, by [4, Corollary 1.13], we have

$$
H^{0}\left(Z, \Omega_{Z}^{1}\right) \cong \bigoplus_{k \in G} H^{1}\left(\mathbb{P}^{2}, \mathcal{J}\left(\mathbb{P}^{2}, \sum_{j=1}^{r}\left\{\frac{k m_{j}}{d}\right\} C_{j}\right)\left(\sum_{j=1}^{r}\left\{\frac{k m_{j}}{d}\right\} d_{j}-3\right)\right) .
$$

In the decomposition (4.8), look at the direct summand corresponding to $k=0$. They are nothing but $H^{1}\left(\mathbb{P}^{2}, \mathcal{O}_{\mathbb{P}^{2}}(-3)\right)=H^{1}\left(\mathbb{P}^{2}, \omega_{\mathbb{P}^{2}}\right)$. By the Serre duality, $\operatorname{dim}_{\mathbb{C}} H^{1}\left(\mathbb{P}^{2}, \omega_{\mathbb{P}^{2}}\right)=\operatorname{dim}_{\mathbb{C}} H^{1}\left(\mathbb{P}^{2}, \mathcal{O}_{\mathbb{P}^{2}}\right)=0$. Therefore, from (4.5), (4.6), (4.7) and (4.8), we get

$$
\sum_{k \in G \backslash\{0\}} h^{0}\left(\Omega_{Y}^{1}(\log E) \otimes \mathcal{L}^{(k)^{-1}}\right)=\sum_{k \in G \backslash\{0\}} \ell_{k} .
$$

Repeating the proof of [19, Proposition 4.6] and using (4.2) we obtain that

$$
h^{0}\left(\Omega_{Y}^{1}(\log E) \otimes \mathcal{L}^{(k)^{-1}}\right) \geq \ell_{d-k},
$$

for $k \in G \backslash\{0\}$. This together with (4.9) means the equality $h^{0}\left(\Omega_{Y}^{1}(\log E) \otimes \mathcal{L}^{(k)^{-1}}\right)=\ell_{d-k}$, thus (4.3) is proved.

4.2. A formula for the monodromy zeta function. By definition, the monodromy zeta function the homogeneous singularity $f\left(x_{0}, \ldots, x_{n}\right)$ at the origin $O$ of $\mathbb{C}^{n+1}$ is the function

$$
\zeta_{f, O}(t)=\prod_{l \geq 0} \operatorname{det}\left(\mathrm{Id}-\left.t T\right|_{H^{l}(M, \mathbb{C})}\right)^{(-1)^{l+1}} .
$$

This function may be expressed via the polynomials $\Delta_{l}(t)$ as $\zeta_{f, O}(t)=\prod_{l \geq 0}\left(t^{\operatorname{dim}_{\mathbb{C}} H^{l}(M, \mathbb{C})} \Delta_{l}\left(\frac{1}{t}\right)\right)^{(-1)^{l+1}}$, from which, by Lemma 4.2 .

$$
\zeta_{f, O}(t)=\prod_{k \in G}\left(1-e^{\frac{2 \pi i k m}{d}} t^{m}\right)^{\sum_{l \geq 0}(-1)^{l+1} h_{l}^{(k)}} .
$$

As explained in [5], the only numbers $\alpha \in(0, n+1) \cap \mathbb{Q}$ such that $n_{\alpha, O}(f)$, the coefficients of $t^{\alpha}$ in $\operatorname{Sp}(f, O)$, can be nonzero are of the form $\frac{k}{d}+p$, with $k, p \in \mathbb{Z}, 1 \leq k \leq d$ and $0 \leq p \leq n+1$. Then it implies from (2.2) and Lemma 3.5 that

$$
n_{\frac{k}{d}+p, O}(f)=\sum_{j \in \mathbb{Z}}(-1)^{j} \operatorname{dim}_{\mathbb{C}} G r_{F}^{n-p} H^{n+j}\left(U, \mathcal{V}_{k}\right),
$$


for integers $1 \leq k \leq d$ and $0 \leq p \leq n+1$, where $\mathcal{V}_{k}$ is the local system corresponding to the element $\left(\mathcal{O}_{\mathbb{P}^{2}}\left(\sum_{j=1}^{r}\left\{\frac{k m_{j}}{d}\right\} d_{j}\right),\left(\left\{\frac{k m_{1}}{d}\right\}, \ldots,\left\{\frac{k m_{r}}{d}\right\}\right)\right)$ in $\operatorname{Pic}^{\tau}(X, D)$ via the isomorphism $R H$ in Theorem 3.1 (cf. Lemma 3.6). Note that $\mathcal{V}_{d}=\mathcal{V}_{0}$. By Proposition 3.8 and (4.11), we have

$$
n_{\frac{d-k}{d}+p, O}(f)=\sum_{j \in \mathbb{Z}}(-1)^{j} h^{p+j}\left(\Omega_{Y}^{n-p}(\log E) \otimes \mathcal{L}^{(k)^{-1}}\right),
$$

for $k \in G$ when $p<n$, and $k \in G \backslash\{0\}$ when $p=n$, where $\mathcal{L}^{(k)}$ and $h^{q}\left(\Omega_{Y}^{p}(\log E) \otimes \mathcal{L}^{(k)^{-1}}\right)$ are as in Lemma 4.1 (see also (3.3)).

Theorem 4.4. The monodromy zeta function and the Hodge spectrum of the singularity $f$ are fit into a relation as follows

$$
\zeta_{f, O}(t)^{(-1)^{n+1}}=\left(1-t^{m}\right)^{1+\sum_{p=1}^{n} n_{p, O}(f)} \prod_{k \in G \backslash\{0\}}\left(1-e^{\frac{2 \pi i k m}{d}} t^{m}\right)^{\sum_{p=0}^{n} \frac{n_{d-k}+p, O}{d}(f)} .
$$

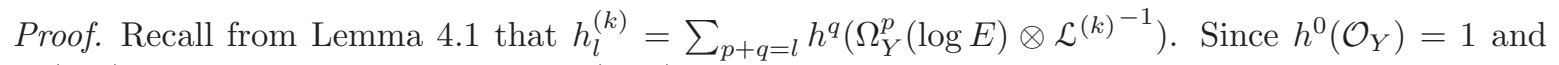
$h^{q}\left(\mathcal{O}_{Y}\right)=0$ for all $q \geq 1$, the formula (4.12) gives

$$
(-1)^{n+1}+(-1)^{n+1} \sum_{p=0}^{n-1} n_{p+1, O}(f)=\sum_{j \in \mathbb{Z}}(-1)^{n+j+1} h_{n+j}^{(0)} .
$$

As in the proof of Lemma 4.2 if $k \in G \backslash\{0\}$, then $h^{0}\left(\mathcal{L}^{(k)^{-1}}\right)=0$, thus by (4.12) we have

$$
(-1)^{n+1} \sum_{p=0}^{n} n_{\frac{d-k}{d}+p, O}(f)=\sum_{j \in \mathbb{Z}}(-1)^{n+j+1} h_{n+j}^{(k)} .
$$

Now applying (4.10) we obtain the statement of the theorem.

Remark 4.5. The formula (4.12) has the following interesting consequence. Assume that $f\left(x_{0}, \ldots, x_{n}\right)$ is a homogeneous polynomial and has isolated singularity at the origin $O$ of $\mathbb{C}^{n+1}$. Then the non-trivial characteristic polynomials of the singularity only appear in the degrees 0 and $n$. This means that $h_{l}^{(k)}=0$ for all $l \notin\{0, n\}$ and $0 \leq k \leq d-1$. Using the proof of Theorem 4.4 we obtain the identities

$$
h_{n}^{(0)}=\sum_{p=1}^{n} n_{p, O}(f) \quad \text { and } \quad h_{n}^{(k)}=\sum_{p=0}^{n} n_{\frac{d-k}{d}+p, O}(f) \text { for } 1 \leq k \leq d-1,
$$

which prove the below result. By convention, we may consider the zero space $\{0\}$ as an eigenspace of the monodromy of the singularity with dimension zero.

Corollary 4.6. Let $f\left(x_{0}, \ldots, x_{n}\right) \in \mathbb{C}\left[x_{0}, \ldots, x_{n}\right]$ be a homogeneous polynomial defining an isolated singularity at the origin $O$ of $\mathbb{C}^{n+1}$. Then the complex dimension of the eigenspace of the monodromy of the singularity with respect to the eigenvalue 1 (resp. $e^{\frac{2 \pi i k}{d}}$, for $1 \leq k \leq d-1$ ) is $\sum_{p=1}^{n} n_{p, O}(f)$ (resp. $\sum_{p=0}^{n} n_{\frac{d-k}{d}+p, O}(f)$, for $1 \leq k \leq d-1$ ).

Acknowledgement. The second and third authors thank Vietnam Institute for Advanced Study in Mathematics and Department of Mathematics - KU Leuven for warm hospitality during their visits. The third author is grateful to Nero Budur for valuable discussions.

\section{REFERENCES}

[1] D. Abramovich and J. Wang, Equivariant resolution of singularities in characteristic 0, Math. Res. Lett. 4 (no. 2-3) (1997), 427-433.

[2] N. Budur, On Hodge spectrum and multiplier ideals, Math. Ann. 327 (2003), no. 2, 257-270.

[3] N. Budur, Multiplier ideals, Milnor fibers, and other singularity invariants, Lecture notes, Luminy, January 2011.

[4] N. Budur, Unitary local systems, multiplier ideals, and polynomial periodicity of Hodge numbers, Adv. Math. 221 (2009), no. 1, 217-250.

[5] N. Budur, Hodge spectrum of hyperplane arrangements, arXiv:0809.3443 
[6] N. Budur and M. Saito, Multiplier ideals, V-filtration, and spectrum, J. Algebraic Geom. 14 (2005), 269-282.

[7] J.P. Demailly, A numerical criterion for very ample line bundles, J. Differential Geom. 37 (1993), no. 2, 323-374.

[8] J. Denef and F. Loeser, Motivic Igusa zeta functions, J. Algebraic Geom. 7 (1998), 505-537.

[9] A. Dimca, Singularities and Topology of Hypersurfaces, Universitext, Springer-Verlag, New York 1992.

[10] L. Ein and R. Lazarsfeld, Global generation of pluricanonical and adjoint linear series on smooth projective threefolds, J. Amer. Math. Soc. 6 (1993), no. 4, 875-903.

[11] H. Esnault, Fibre de Milnor d'un cône sur une courbe plane singulilarité, Invent. Math. 68 (1982), 477-496.

[12] H. Esnault and E. Viehweg, Lectures on vanishing theorem, DMV Sem, vol. 68, Birkhäuser, Basel, 1992.

[13] J. Howald, Multiplier ideals of monomial ideals, Trans. Amer. Math. Soc. 353 (2001), 2665-2671.

[14] J. Kollár, Singularities of Pairs, Proc. Sympos. Pure Math. A.M.S. 62, Part 1 (1997), 221-287.

[15] R. Lazarsfeld, Positivity in Algebraic Geometry II - Positivity for Vector Bundles, and Multiplier Ideals, SpringerVerlag, Berlin 2004.

[16] D.T. Lê, Some remarks on relative monodromy. In P. Holm, editor, Real and complex singularities (Proc. Ninth Nordic Summer School/NAVF Sympos. Math., Oslo, August 5-25, 1976), pages 397-403. Sijthoff and Noordhoff, Alphen aan den Rijn, 1977.

[17] A. Libgober, Alexander polynomial of plane algebraic curves and cyclic multiple planes, Duke Math. J. 49 (1982), 833-851.

[18] A. Libgober, Alexander invariants of plane albebraic curves, Proc. of Sym. in Pure Math. 40 (1983), 135-143.

[19] F. Loeser and M. Vaquié, Le polynôme d'Alexander d'une courbe plane projective, Topology 29 (1990), 163-173.

[20] J. Milnor, Singular points of complex hypersurfaces, Ann. Math. Studies 61, Princeton Univ. Press 1968.

[21] J. Milnor and P. Orlik, Isolated singularities defined by weighted homogeneous polynomials, Topology 9 (1970), 385-393.

[22] M. Mustaţă, Singularities of pairs via jet schemes, J. Amer. Math. Soc. 15 (2002), 599-615.

[23] A. Nadel, Multiplier ideal sheaves and Kähler-Einstein metrics of positive scalar curvature, Ann. of Math. (2) 132 (1990), no. 3, 549-596.

[24] R. Randell, Milnor fibers and Alexander polynomials of plane curves, Proc. of Sym. in Pure Math. 40 (1983), $415-419$

[25] M. Saito, Mixed Hodge modules and applications, Proceedings of the ICM Kyoto, 1991, 725-734.

[26] J.H.M. Steenbrink, Mixed Hodge structure on the vanishing cohomology, Real and complex singularities, Oslo 1976, Sijthoff and Noordhoff, Alphen aan den Rijn, 1977, 525-563.

[27] J.H.M. Steenbrink, Intersection form for quasi-homogeneous singularities, Compositio Math. 34 (1977), 211-223.

[28] A.N. Varchenko, Asymtotic Hodge structure on vanishing cohomology, Izv. Akad. Nauk SSSR Ser. Mat. 45 (1981), $540-591$.

Department of Mathematics, Vietnam National University

334 Nguyen Trai Street, Thanh Xuan District, Hanoi, Vietnam

E-mail address: leqthuong@gmail.com

BCAM - Basque Center for Applied Mathematics

Alameda de Mazarredo 14, E-48009 Bilbao, Basque Country, Spain

E-mail address: qle@bcamath.org

Department of Mathematics, Vietnam national University

334 Nguyen Trai Street, Thanh Xuan District, Hanoi, Vietnam

E-mail address: nphlan@gmail.com

Department of Mathematics, Vietnam National University

334 Nguyen Trai Street, Thanh Xuan District, Hanoi, Vietnam

E-mail address: phoductai@gmail.com 